\title{
Neural stem cells attacked by Zika virus
}

\author{
Cell Research (2016) 26:753-754. doi:10.1038/cr.2016.68; published online 10 June 2016
}

The current outbreak of Zika virus-associated diseases in South America and its threat to spread to other parts of the world has emerged as a global health emergency. Insights from cell and animal models to understand how Zika virus causes severe birth defects may lead to treatments and prevention of these diseases.

Zika virus (ZIKV) is a positive single-stranded RNA flavivirus and is primarily spread by the bite of an infected Aedes species mosquito. ZIKV was first discovered in 1947 in Africa and, perhaps due to the low number of documented symptomatic cases, study of Zika virus disease had been largely neglected. The recent ZIKV outbreak in South America, especially in Brazil, and the coincidental increase in cases of microcephaly led the World Health Organization (WHO) to declare a public health emergency of international concern [1]. It has ignited tremendous interest and increased efforts by scientists in an attempt to understand, treat and prevent the spread of ZIKV. In the past few months alone, it has been shown that ZIKV infection could lead to brain abnormalities in the human fetus and that ZIKV preferentially targets human neuronal precursor cells in both monolayer and cortical brain organoid culture systems, and stunts their growth [2-4]. These and other findings have helped the US Centers for Disease Control and Prevention (CDC) to conclude that Zika virus causes microcephaly. However, it is still not clear how the ZIKV wreaks havoc on developing fetuses and causes severe brain defects in vivo. To address this question, $\mathrm{Wu}$ and colleagues, in a recent study published in Cell Research, developed a new mouse model that recapitulates effects of ZIKV infection and elucidates how it may cause brain malformations and microcephaly [5].

First, to determine if ZIKV can infect brain cells, Wu et al. injected virus directly into the lateral ventricle of fetal mouse brains at embryonic day 13.5 (E13.5), a stage with prominent cortical neurogenesis, and analyzed viral infection at E17.5 and immediately after birth. They observed strong signals of viral infection in the dorsal telencephalon, which gives rise to the cerebral cortex, but also in the nearby striatum. Because ZIKV is usually contracted from a mosquito bite and suspected to cross the fetal-placental barrier, they tested a vertical transmission model by injecting the ZIKV into the abdominal cavity of pregnant mice, again at E13.5 (Figure 1). They detected the presence of virus in the blood of pregnant mice at E14.5, and ZIKV-specific RNA in the placenta at E16.5. Interestingly, the virus homed to and infected the dorsal telencephalon, whereas other regions of the brain, including the striatum, were spared. Upon more detailed analysis, they found that the virus infected mostly radial glial cells (RGCs), which are cortical neural progenitors localized in the ventricular zone (VZ) of the dorsal cortex, a finding consistent with recent in vitro studies using human neural progenitor cells.

Second, Wu et al. investigated the impact of ZIKV infection in the fetal brain. They discovered that cells in the $\mathrm{VZ}$, the sub-ventricular zone (SVZ) and intermediate zone (IZ), did not divide as much as they normally do. Consequently, the layer thickness of VZ and SVZ of ZIKV-infected fetal brains was significantly reduced compared to uninfected control brains. Moreover, the number of intermediate progenitors was also significantly reduced.

To provide a possible mechanism as to why ZIKV is causing neuronal progenitors to divide less frequently than usual, Wu et al. performed quantitative real-time PCR to measure the level of messenger RNA of a number of genes known to be associated with primary microcephaly in humans. They identified many genes related to regulation of the cell cycle, including Microcephalin, $A S P M$ and $C D K 6$, to name a few, that were significantly reduced in ZIKVinfected fetal brains. Interestingly, they noticed a marked increase in the expression of interleukin-17a, a gene involved in the IL-17 pathway of the immune response system. Notably, a previous study in mice had shown that maternal activation of the IL-17 pathway can induce autism-like behaviors and promote abnormal cortical development in offspring [6]. Therefore, ZIKV infection might have a non-cell autonomous impact on the developing nervous system.

Finally, Wu et al. investigated whether other regions of the cerebral cortex were affected by ZIKV infection. They found the outer length of the infected cortex was shorter than normal, despite no significant differences in thickness of the upper cortical layers. Notably, they also found smaller lateral ventricle cavities with reduced ventricular surfaces in ZIKV-infected fetal brains. This is somewhat different from enlarged ventricles found in aborted fetuses with known ZIKV infection [2].

The study described here by $\mathrm{Wu}$ 


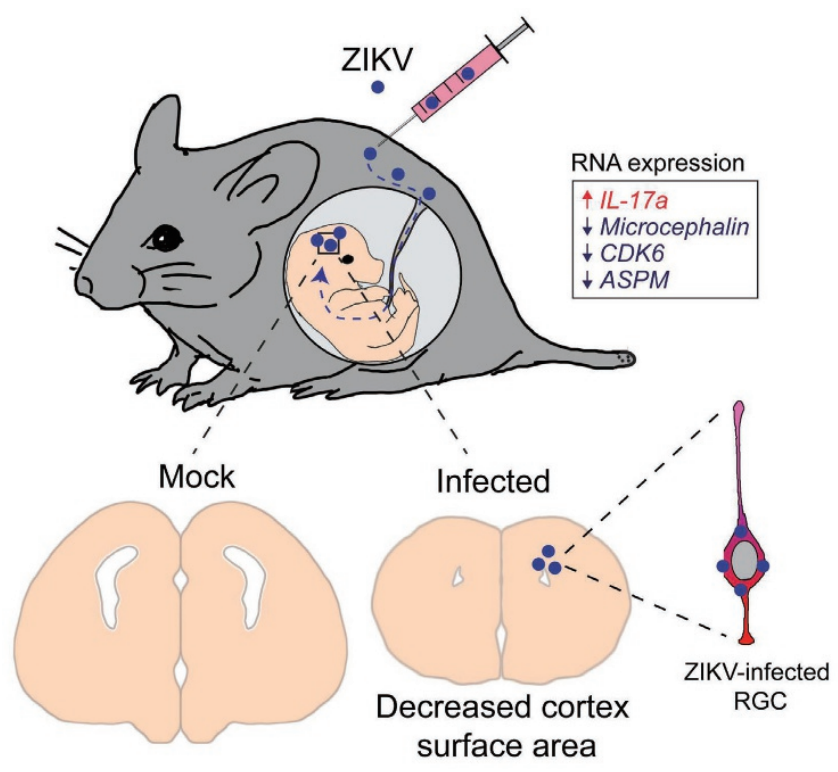

Figure $1 \mathrm{~A}$ mouse model of Zika virus (ZIKV) vertical transmission. ZIKV (blue dots) injected into the abdominal cavity of a pregnant mouse, crosses the fetal-placental and homes to the dorsal cortex of fetal brain (dashed line with arrowhead). The ZIKV infects radial glial cells (RGCs) located in the ventricular zone of the dorsal cortex and inhibits normal cell division. Together with reduced cell proliferation, decreased expression of genes related to cell cycle and increased immune activation through upregulation of IL-17a (see box), ZIKV-infected fetal brains develop abnormally and resemble microcephaly that is observed in humans.

and colleagues, corroborated by other recent studies from several independent groups, have unequivocally demonstrated the potency of ZIKV and its devastating effects on the development of fetal brains [7-10]. It has become apparent that ZIKV readily crosses the fetal-placental barrier, directly targets neuronal progenitors, either by killing them or attenuating their proliferation, and causes brain defects and microcephaly in both animal models and in humans. These animal models will serve as a valuable platform for drug and compound testing to prevent and treat Zika virus-related diseases. Looking ahead, these models can also be used to develop effective ZIKV vaccines to combat and eradicate the threat that is looming over the entire global population.

Ha Nam Nguyen ${ }^{1,2}$, Xuyu Qian
Hongjun Song
1,2,

${ }^{1}$ Institute for Cell Engineering, ${ }^{2}$ Department of Neurology, ${ }^{3}$ Biomedical Engineering Graduate Program, ${ }^{4}$ The Solomon Snyder Department of Neuroscience, 'Department of Psychiatry and Behavioral Sciences, Johns Hopkins University School of Medicine, Baltimore, MD 21205, USA. Correspondence: Guo-li Ming

E-mail: gming1@jhmi.edu

\section{References}

1 Heymann DL, Hodgson A, Sall AA, et al. Lancet 2016; 387:719-721.

2 Driggers RW, Ho CY, Korhonen EM, et al. N Engl J Med 2016; 374:2142-2151.

3 Qian X, Nguyen HN, Song MM, et al. Cell 2016; 165:1238-1254.

4 Tang H, Hammack C, Ogden SC, et al. Cell Stem Cell 2016; 18:587-590.

5 Wu KY, Zuo GL, Li XF, et al. Cell Res 2016; 26:645-654

6 Choi GB, Yim YS, Wong H, et al. Science 2016; 351:933-939.

7 Cugola FR, Fernandes IR, Russo FB, et al. Nature 2016; 534:267-271.

8 Lazear HM, Govero J, Smith AM, et al. Cell Host Microbe 2016; 19:720-730.

9 Li C, Xu D, Ye Q, et al. Cell Stem Cell 2016 May 9. doi:10.1016/j.stem.2016.04.017

10 Miner JJ, Cao B, Govero J, et al. Cell 2016; 165:1081-1091. 